\title{
Nucleotide sequence of the genes coding for minor fimbrial subunits of the F1C fimbriae of Escherichia coli
}

\author{
I. van Die ${ }^{(1)}\left(^{*}\right)$, C. Kramer (1), J. Hacker ${ }^{(2)}$, H. Bergmans ${ }^{(1)}$, \\ W. Jongen ${ }^{(1)}$ and W. Hoekstra ${ }^{(1)}$ \\ (1) Dept. of Molecular Cell Biology, University of Utrecht, \\ Padualaan 8, CH 3584 Utrecht (the Netherlands) and

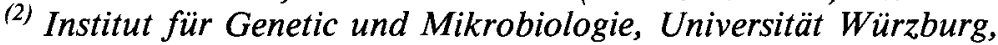 \\ Röntgenring 11, D-8700 Würzburg (Germany)
}

\begin{abstract}
SUMMARY
F1C fimbriae allow uropathogenic Escherichia coli to adhere to specific epithelial surfaces. This adhesive property is probably due to the presence of minor fimbrial components in F1C fimbriae. The foc gene cluster encoding F1C fimbriae has been cloned, as described previously. Here we present the nucleotide sequence $(2081 \mathrm{bp}$ ) coding for the F1C minor fimbrial subunits. The structural genes code for polypeptides of 175 (FocF), 166 (FocG), and 300 (FocH) amino acids.

The deduced amino acids of the $\mathrm{F} 1 \mathrm{C}$ minor subunits were compared with the reported sequences of the minor subunits of other types of fimbriae. The data show that the Foc minor subunits are highly homologous to the corresponding Sfa proteins, whereas homology to the minor subunits of type 1 and $P$ fimbriae is much lower.
\end{abstract}

Key-words: Pilus, Escherichia coli, Adherence, Urinary tract; Foc protein, Minor subunits, Sequencing, Homology.

\section{INTRODUCTION}

Virulence of uropathogenic Escherichia coli strains has been related to their ability to adhere to uroepithelial cells. Adherence is mediated by adhesive proteins (minor subunits) that are often located at the tip of fimbriae (Klemm, 1985). In uropathogenic $E$. coli, various fimbrial adhesins (e.g. type $1, \mathrm{P}, \mathrm{S}$, and $\mathrm{F} 1 \mathrm{C}$ ) are found that are distinguished by their receptor specificities (Orskov and Orskov, 1983; Klemm, 1985; Hacker, 1989). It has been shown that F1C fimbriae contribute to the adhesive properties of uropathogenic E. coli. Virkola et al. (1988) showed that F1C fimbriae mediate adherence to the collecting ducts and the distal tubules of the human kidney. Recently, Marre et al. (1990)

Submitted January 12, 1991, accepted March 15, 1991.

$\left({ }^{*}\right)$ Corresponding author. Present address: Dept. of Medical Chemistry, Vrije Universiteit, Van der Boechorststraat 7, 1081 BT Amsterdam (the Netherlands). 
showed the adherence of F1C fimbriae to cultured renal tubulus cells.

The gene cluster encoding F1C fimbriae has been cloned and analysed in detail (Van Die et al., 1985; Riegman et al., 1990). Genetically, F1C fimbriae have been shown to be very closely related to S fimbriae (Ott et al., 1987, 1988; Riegman et al., 1990). In both gene clusters, the distal part encodes minor fimbrial subunits (Hacker, 1989; Schmoll et al., 1989; Riegman et al., 1990). It is expected that (one of) the minor subunits will confer the adhesive properties to the F1C fimbriae, as has been been shown for other fimbrial types (Lindberg et al., 1986; Klemm and Christiansen, 1987; Morschhäuser et al., 1990). For the $\mathrm{S}$ fimbriae, the $15-\mathrm{kDa}$ protein SfaS has been determined as the component that binds to carbohydrate chains terminating with sialyl $\alpha 2 \rightarrow 3 \mathrm{Gal}-\beta 1 \rightarrow \mathrm{R}$ (Parkinen et al., 1986; Moch et al., 1987; Morschhäuser et al., 1990). In this paper, we present the nucleotide sequence of the region coding for minor fimbrial components of the F1C fimbriae. This sequence is compared with the published nucleotide sequence of the corresponding region of the S-fimbrial gene cluster.

\section{MATERIALS AND METHODS}

\section{Bacterial strains and phages}

The E. coli K12 strain JM101 was used to propagate phages $\mathrm{M} 13 \mathrm{mp} 8$, $\mathrm{mp} 9$ and clones derived from these strains (Messing and Vieira, 1982).

\section{Recombinant DNA techniques}

Restriction endonucleases and ligase (Pharmacia, Sweden) were used according to the manufacturers' specifications. Isolation of plasmid or phage RF DNA was carried out by the mini-lysate method, essentially as described by Holmes and Quigley (1981). Isolation of single-stranded M13 DNA was performed essentially according to Heidecker et al. (1980). Nucleotide sequencing was carried out by the chain termination method of Sanger et al. (1977). With the aid of a T7-sequence kit (Pharmacia), recombinant M13 or plasmid DNA was sequenced according to the protocol supplied by the manufac- turer. Primers used were the M13 primer supplied with the kit, or alternatively 18-mer oligodeoxynucleotides synthesized on a "Biosearch 8600 " DNA synthesizer.

Transformation was carried out essentially as described by Mandel and Higa (1970).

\section{RESULTS AND DISCUSSION}

\section{Nucleotide sequence of the focF, focG and focH genes}

The approximate localization of the foc $\mathrm{F}$, foc $\mathrm{G}$ and $f o c \mathrm{H}$ genes, encoding minor fimbrial subunits, in the F1C gene cluster has been described previously (Riegman et al., 1990). The strategy for the determination of the nucleotide sequence is outlined in figure 1 . Restriction frag-

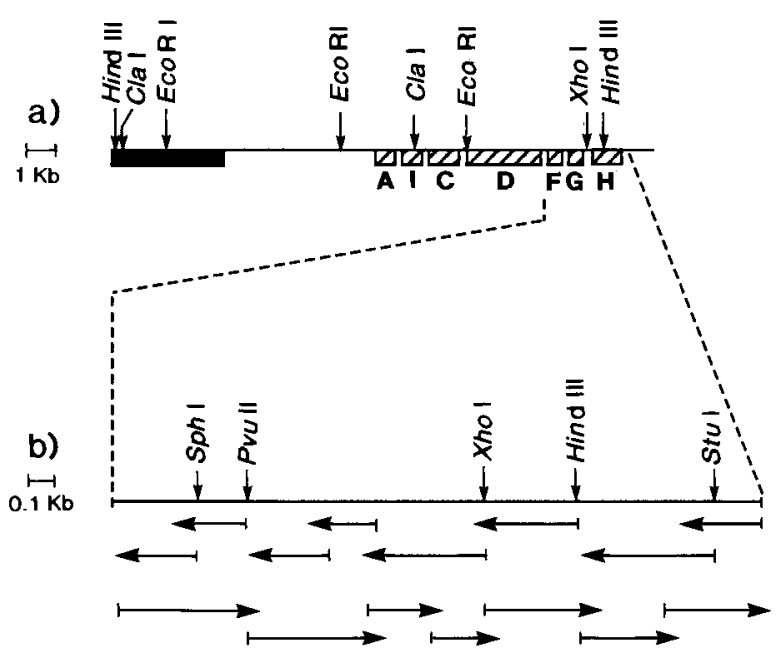

Fig. 1. Physical and genetic map of the foc gene cluster and sequencing strategy.

(a) Physical and genetic map of pPIL110-51 (Van Die et al., 1985; Riegman et al., 1990); the hatched boxes represent genes A-I, involved in the expression of F1C fimbriae; the black box represents pACYC184 (Chang and Cohen, 1978) vector DNA.

(b) Strategy of nucleotide sequence analysis. The length of the sequenced DNA fragments and the direction of sequencing is shown by arrows. 
ments of pPIL110-51 were cloned in M13 mp8 or mp9 and sequenced. A nucleotide sequence of 2081 bp was determined by sequencing both strands of the entire region, and is shown in figure 2 . Since the amino acid sequences of the F1C minor subunit proteins have not been elucidated, it is not possible to precisely locate the $\mathrm{N}$ termini. However, three open-reading frames (ORF) became apparent from the nucleotide sequence. The first ORF (focF) starts at an ATG codon at position 31 , the second and third ORF at positions 579 and 1142, respectively. The first part of the sequences show the characteristics of prokaryotic signal sequences, with potential cleavage sites as indicated in figure 2. The molecular weights, calculated on the basis of the sequence data, of the proteins encoded by these genes are 15, 17 and $30 \mathrm{kDa}$, respectively. Sodium dodecyl sulphatepolyacrylamide gel electrophoresis (SDS-PAGE) analysis of F1C fimbriae showed, next to the 16-kDa FocA major subunit, two subunits with apparent molecular weight of 14 and $32 \mathrm{kDa}$, which correspond well with the calculated masses for FocG and FocH (Riegman et al., 1990). No $17-\mathrm{kDa}$ protein has been observed by SDSPAGE analysis of F1C fimbriae. This might be explained by comigration of the putative foc $\mathrm{F}$ product with the abundant $16-\mathrm{kDa}$ FocA protein.
ACGgCTAgITgCaggtaAagaggCAgGga ATG GTG Aag GAT ATT ATT FocF: Met Val Lys ABp Ile Ile AAA ACA GTG ACA TTC TCC TGC ATG CTG GCC GGC AGT ATG TTC GTT ACC
Lys Thr Val Thr Phe Ser CYs Met Leu Ala GlY Ser Met phe Val Thr TGT CAT GTC TGT GCA GCG GGT TCT GTG GTG AAT ATT ACA GGC AAT GTT 144 Thr Gly Asn Val CAG Gat AAC ACC TGC GAT GTT GAC ATT AAC TCC CGA AAC CTT GAT GTC
Gln Asp Agn Thr Cys Agp Val Asp Ile Asn Ser Arg Asn Leu Asp Val AGT CTG GGA AGT TAT GAC AGC CGA CAG TTT ACC GCA GCT GGT GAT ACC 240 Ser Leu Gly Ser Tyr Agp Ser Arg Gln phe Thr Ala Ala Gly Asp Thr ACA CCT GCG TCG GTA TTT CAT GTC GGG TTA ACC TCC TGC GGC AGT GCT
Thr PIo Ala Ser Val phe His Val Gly Leu Thr Ser Cys Gly Ser Ala GTC AGT GCA GTG Aag CTG ACA TTT ACG GGC ACA CCA GAT Aat CAG GAG 336 Val Ser Ala val Lys Leu Thr phe Thr Gly Thr Pro Asp Asn Gln Glu GCG GGG CTT ATT CAG ATT AAC AGC ATA AAT GGA GCG CGG GGT GTG GGG Ala Gly Leu Ile Gln Ile Asn Ser Ile Asn Gly Ala Arg Gly val Gly ATT CAg CTT CTT Gat aAg Gat aAa CAT Gag CTg AaA ATT Aat GTG CCG 432 Ile Gln Leu leu Asp Lys Asp Lys his Glu Leu Lys Ile Asn val pro ACA ACA ATT GCG rTIA ATG CCG GGA ACA CAG ACC ATA GCG TTT TAT GCC
Thr Thr Ile Ala Leu Met Pro Gly Thr Gln Thr Ile Ala phe Tyr Ala CGT CTG AAA GCC ACT TAT CTT CCG GTA AAG GCC GGT AAT GTT GAT GCG 528 Arg Leu Lys Ala Thr Tyr Leu Pro val Lys ala Gly Asn val Asp Ala GTG ATT Aat TTt GTa CTT GaC tat cag taAatataAcacagaggaaAacag Val Ile Asn the val Leu Asp Tyr Gln End FocG: ATC AAA CTG AAA GCT ATT ATA TTG GCC ACC GGT CTT ATT AAC TGT ATT 627
Met Lys Leu Lys Ala Ile Ile Leu Ala Thr GLy Leu Ile Asn Cys Ile GCA TTT TCA GCA CAG GCA GTG GAT ACG ACG ATT ACT GTT ACA GGG AGG Ala the Ser Ala Gln Alafval Asp Thr Thr Ile Thr val Thr Gly Arg GTA TTG CCA CGT ACC TGT ACC ATP GGT AAT GGA GGA AAC CCA AAC GCC 723 Val Leu Pro Arg Thr Cys thr Ile Gly Asn Gly Gly Asn Pro Asn Ala ACC GTT GTT TTG GAT AAC GCT TAC ACT TCT GAC CTG ATA GCA GCC AAC
Thr Yal Val Leu Asp Asn Ala TYr Thr Ser Asp Leu Ile Ala Ala Asn AGC ACC TCT CAG TGG AaA AAT TTT TCG TTG ACA TTG aCG Aat TGT CAG 819 Aan $C_{Y} \mathrm{G}$ Gln AAT GTA AAC AAT GTT ACT AGC TTT GGT GGA ACC GCA GAA AAT ACA AAT Asn Val Asn Asn Val Thr Ser Phe Gly Gly Thr Ala Glu Asn Thr Aan
TAT TAC AGA AAT ACA GGG GAT GCT ACT AAT ATC ATG GTT GAG CTA CAG 915 TAT TAC AGA AAT ACA GGG GAT GCT ACT AAT ATC ATG GTT GAG CTA CAG 915
Tyr Tyr Arg Agn Thr Gly Asp Ala Thr Asn Ile Met val Glu Leu Gln GAA CAA GGT AAT GGT AAT ACC CCC TPT AAA GTT GGT TCA ACA AAA GTT GTT ACA GTG AGC AAT GGG CAG GCG ACA TTC AAT CTT AM GTC CGT GCC 1011 GTT ACA GTG AGC AAT GGG CAG GCG ACA TTC AAT CTT AMA GTC CGT GCC
Val Thr Val Ser Aan GlY GIn Ala Thr Phe Aan Leu LY" val Arg Ala GTA AGC AAA GGT AAT GCT GGT GCG GGA AGT ATT AAT TCA CAA ATT ACT
Val Ser LyB Gly Asn Ala Gly Ala Gly Ser Ile Asn Ser Gln Ile Thr
GTC ACC TAT ACC TAT GCG TAa ATATTATCCCCTCTTPAAGAAAAGCACCGTCCTCG $\$ 115$

agGGCTGTGTTPTATTCACATTTATA ATG GCA TAT TCC CAG CCA TCG TTT GCA
Foch: Met Ala Tyr Ser Gln Pro Ser phe Ala CTG TTG TGC AGA AAT AAC CAG ACA GGG CAG ACT TTT CAG TCT GGA GAC 1216 Leu Leu Cys arg Asn Asn Gln Thr Gly Gln Thr Phe Gln SerfGly Asp AGT CGC TTC AAT ATC ACT CTT TCC CCA ACA GTT CAG TAT GAT AAA GCC ATT ACA GTT CTG GAT TTA AAT CAA CTG GTG TTA TGT CAG AAT GAA GAT 1312 ATT ACA GTT CTG GAT TTA AAT CAA CTG GTG TTA TGT CAG AAT GAA GAT
Ile Thr val Leu Asp Leu Asn Gln Leu val Leu Cys Gln Agn Glu Asp GCC TCT GGT CAG AAC TAT GAC TAT CTC AGG GTC AGA CAG GGA ACC GGT
hla Ser Gly Gln Asn TYr Asp Tyr Leu AIg Val AIg Gln Gly Thr Gly TTT TCT CCT TCA TTA GAT GCT AAA ACA TAC GGA AGG CTG GAC TTT ACA 1408
Phe Ser Pro Ser Leu Asp Ala LY Thr Tyr Gly AIg Leu Asp phe Thr AaC AGg CIT TCT GGg TAT AGT CAG ACG TTA CCG CTG CAG CAA GAC ACA Asn Arg Leu Ser Gly Tyr Ser Gln Thr Leu Pro Leu Gln Gln Asp Thr AAG CCA ACA GAA GCT TAC TGG CAA TAT GGT GTA TGG AAA CCT TTC CCG 1504 GCA AAA ATG TAC CTT TAT CCT GAG CCG GGC GTT TTC GGG AAA CTG ATA ala Lys met Tyr Leu tyr pro Glu Pro Gly val phe Gly Lys Leu Ile CAT GCC GGA GAA TTIA GTG GCC ACA GTT TAT GTT AAT AAG TTT TCC ACC 1600 Hia Ala Gly Glu Leu val Ala Thr val Tyr val Asn Lys phe Ser Thr ATG GGG CAG GAG GCA GGG GAG AGA AAT TTC ACC TGG CGT TTC TAT GCA ACG AAT GAT GTC TAT ATC CAG ACA GGT ACA TGC AGG GTC TCA TCG AAC 1696
Thr Asn Asp Val Tyr Ile Gln Thr Gly Thr Cys Arg Val Ser Ser Asn Aat GTC AaA GTT GAC CTT CCG TCC TAT CCC GGA GGC CCG GTA ACA GTC Asn val Lys val Asp Leu pro Ser Tyr pro Gly Gly pro val thr val CCT CTT ACT GTA CGT TGC GAC CAG ACA CAG TCG GTC AGC TAT ACC CTG 1792 pro Leu Thr val Arg Cys Asp Gln Thr Gln Ser val Ser TYr Thr Leu TCA GGT TCT GTA ACA GGA AGT GGT AAT ACT GTA TTC GCA AAT ACG GCA
Ser Gly Ser Val Thr Gly Ser Gly Asn Thr Val phe Ala Asn Thr Ala ACA TCA GGG GCC GGC GGT GTG GGT GTA CAG TTG TCG GAC AAC GCG GGG 1888 Thr Ser Gly Ala Gly Gly Val Gly Val Glin Leu Ser Asp Asn Ala Gly CTG GTP CCG GCC GGA CAA CCG AGG TCT CTG GGA CAG GTA GGC AGC TCT Leu val Pro Ala Gly gln Pro Arg Ser Leu Gly Gln val Gly Ser Ser CCT GTG AGT CTG GGG CTG MAG GCC TCT TAT GCT CTG ACC GGT CAG GCA 1084 Pro val ser Leu Gly Leu Lys Ala Ser Tyr Ala Leu Thr Gly Gln Ala AGT CCG ACG CCC GGT GCT GTC CAG TCA GTG ATA AAT GTG ACT TTT AGC Sor Pro Thr Pro Gly Ala val Gln Ser val Ile Asn Val Thr phe Ser TAC AAC TAG AATGCAGTTGTGGCGGTAATGATATPACTGCTCTTTATAT 2081 Tyr Asn End

Fig. 2. Nucleotide sequence and deduced amino acid sequence of the foc $\mathrm{F}$, foc $\mathrm{G}$, and foc $\mathrm{H}$ gene.

Numbering of the residues starts at the lefthand side of the DNA fragment sequenced (see fig. 1). Numbers refer to the nucleotide positions. Potential signal sequence cleavage sites are indicated by arrows. 


\section{Comparison of the Foc minor subunit proteins with minor proteins of other fimbriae}

The amino acid sequences, deduced from the nucleotide sequences, of the FocF, FocG and FocH proteins were compared with the corresponding proteins of $\mathrm{S}$ (Sfa), type 1 (Fim), and P (Fst) fimbriae (Klemm and Christiansen, 1987; Lund et al., 1985; Schmoll et al., 1989). The results (fig. 3, table I) show that all three Foc proteins show a high degree of similarity to the corresponding Sfa proteins. Homology to the Fim and Fst minor fimbrial proteins is clearly lower.

FocF and $\mathrm{SfaG}$ are nearly identical, with only three amino acid substitutions (homology $98 \%$ ). Also, FocH and SfaH are very homologous (84 \%). Interestingly, homology between FocG and SfaS appears to be much lower $(59 \%)$. SfaS represents the specific adhesin binding to sialic acid (Morschhäuser et al., 1990), whereas F1C fimbriae lack that specific adhesive property. The high degree of homology between the foc and $s f a$ gene clusters was also found in previous studies (Ott et al., 1987, 1988; Riegman et al., 1990; Schmoll et al., in press). It strongly suggests that these gene clusters represent a family of adhesin determinants, as was observed for the $\mathrm{P} / \mathrm{Prs}$ and the type 1 fimbrial adhesins; in the $\mathrm{P} / \mathrm{Prs}$ and type 1 fimbrial systems, adhesive properties have been designated for the large 35-kDa minor subunits (Lindberg et al., 1986; Klemm and Christiansen, 1987; Lund et al., 1988; Riegman et al., 1988). Up until now, it has not been clear which of the F1C subunits is responsible for the observed adhesive properties. It was found that HB101 cells carrying F1C fimbriae lacking the 32-kDa FocH subunit showed

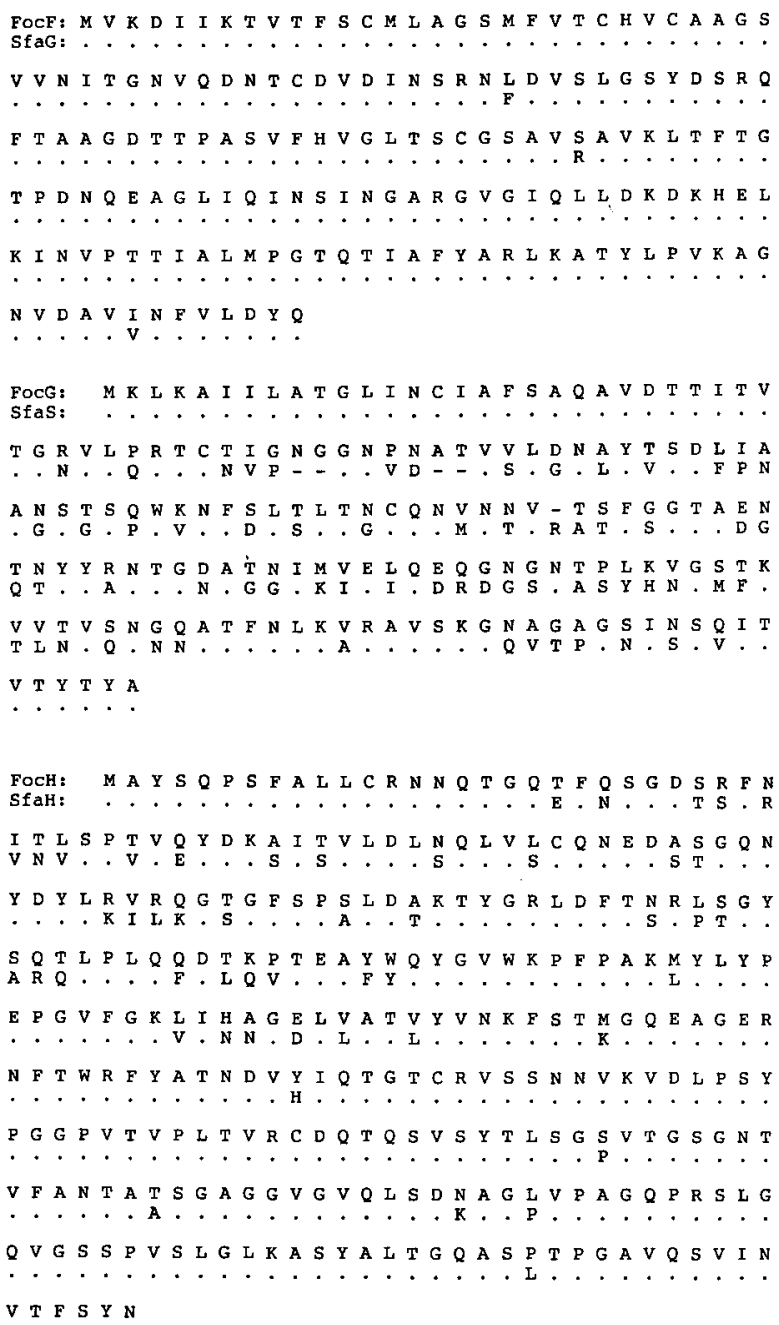

Fig. 3. Comparison of the deduced amino acid sequences (given in standard one-letter code) of FocF, FocG and FocH with the $\mathrm{SfaG}, \mathrm{SfaS}$ and $\mathrm{SfaH}$ minor subunit proteins.

Gaps (-) have been introduced to obtain maximum homology between the proteins. Identical amino acids between Foc and Sfa proteins are indicated as dots in the Sfa sequence.

Table I. Percentage homology between the Foc minor proteins and the corresponding proteins of S (Sfa), type 1 (Fim) and P (Fst) fimbriae (Schmoll et al., 1989; Klemm and Christiansen, 1987; Lund et al., 1985).

\begin{tabular}{lccccccccc} 
& SfaG & SfaS & SfaH & FimF & FimG & FimH & FstE & FstF & FstG \\
\hline FocF & 98 & - & - & 35 & - & - & 23 & $\overline{23}$ & - \\
FocG & - & 59 & $\overline{44}$ & - & 41 & $\overline{34}$ & - & - & 17 \\
FocH & - & - & 84 & - & - & - &
\end{tabular}


normal F1C adhesive properties (Van Die and Korhonen, unpublished results). In receptorbinding studies, Marre et al. (1990) showed that the inhibition profiles of S and F1C fimbriae, although different in the sialic acid-binding property, resemble each other quite well. In addition, it was shown that the SfaG subunit contributes to binding of $\mathrm{S}$ fimbriae to renal tubulus cells. Based on this observation and the very high homology found between FocF and $\mathrm{SfaG}$, the FocF protein most likely mediates the observed F1C adhesion to renal tubulus cells. It might be possible, however, that FocG (or FocH) is also involved in recognition of a still unknown receptor. $\mathrm{P}$ fimbriae (type $\mathrm{F} 7_{1}$ ) have been shown to possess several adhesins: the $35-\mathrm{kDa}$ protein represents the $\mathrm{P}$ adhesin (Riegman et al., 1988), whereas the two small minor subunits FsoE and FsoF are responsible for binding to fibronectin (Westerlund et al., submitted for publication).

The latter results suggest that fimbriae can be regarded as very flexible carriers, able to present several different adhesins. Experiments are in progress to construct defined FocA, FocF and FocG mutants, so as to elucidate the possible adhesive properties of the different Foc fimbrial subunits in detail.

\section{Acknowledgements}

We thank E. van Beurden, L. Stabel, I. van Megen and J. van Oosterhout for technical assistance in part of the work.

\section{Séquence nucléotidique des gènes codant pour les sous-unités des fimbriae F1C de Escherichia coli}

Les fimbriae F1C permettent aux souches de Escherichia coli uropathogènes d'adhérer aux surfaces épithéliales spécifiques. Cette propriété adhésive est probablement due à la présence de composants mineurs des fimbriae F1C. Le groupe de gènes foc codant pour les fimbriae F1C ont été clonés selon des méthodes déjà décrites. Nous présentons ici la séquence nucléotidique $(2081 \mathrm{pb})$ codant pour les sous-unités mineures des fimbriae F1C. Les gènes structuraux codent pour les polypeptides de 175 (FocF), 166 (FocG) et 300 (FocH) acides aminés.
Les séquences d'acides aminés des sous-unités mineures F1C, déduits des séquences nucléotidiques, ont été comparés avec celles connues des séquences des sous-unités mineures d'autres types de fimbriae. Ce travail montre que les sous-unités mineures Foc sont fortement homologues à celles des protéines $\mathrm{Sfa}$, alors que l'homologie avec les sous-unités mineures des fimbriae de type 1 et $\mathbf{P}$ est moins importante.

Mots-clés: Pilus, Adhérence, Escherichia coli, Tractus urinaire; Sous-unités mineures, Séquençage, Protéine Foc, Homologie.

\section{References}

Chang, A.C.Y. \& Cohen, S.N. (1978), Construction and characterization of amplifiable multicopy DNAcloning vehicles derived from the P15A cryptic plasmid. J. Bact., 134, 1141-1156.

Hacker, J. (1989), Genetic determinants coding for fimbriae and adhesins of extraintestinal Escherichia coli. Curr. Top. Microbiol. Immunol., 151, 191-217.

Heidecker, G., Messing, J. \& Gronenborn, B. (1980), A versatile primer for DNA sequencing in the M13mp2-cloning system. Gene, 10, 69-73.

Holmes, D.S. \& Quigley, M. (1981), A rapid boiling method for the preparation of bacterial plasmids. Analyt. Biochem., 114, 193-197.

Inouye, M. \& Halegoua, S. (1980), Secretion and membrane localization of proteins in Escherichia coli. Crit. Rev. Biochem., 7, 339-371.

Klemm, P. (1985), Fimbrial adhesins of Escherichia coli. Rev. infect. Dis., 7, 321-340.

Klemm, P. \& Christiansen, G. (1987), Three fim genes required for the regulation of length and mediation of adhesion of Escherichia coli type 1 fimbriae. Mol. gen. Genetics, 208, 439-445.

Lindberg, F., Lund, B. \& Normark, S. (1986), Gene products specifying adhesion of uropathogenic Escherichia coli are minor components of pili. Proc. nat. Acad. Sci. (Wash.), 83, 1891-1895.

Lund, B., Lindberg, F.P., Baga, M. \& Normark, S. (1985), Globoside-specific adhesins of uropathogenic Escherichia coli are encoded by similar transcomplementable gene clusters. J. Bact., 162, 1293-1301.

Lund, B., Marklund, B.I., Strömberg, N., Lindberg, F., Karlsson, K. \& Normark, S. (1988), Uropathogenic Escherichia coli can express serologically identical pili of different receptor-binding specificities. Mol. Microbiol., 2, 255-263.

Mandel, M. \& Higa, A. (1970), Calcium-dependent bacteriophage DNA infection. J. mol. Biol., 53, 154-162.

Marre, R., Kreft, B. \& Hacker, J. (1990), Genetically engineered $S$ and F1C fimbriae differ in their contribution to adherence of Escherichia coli to cultured renal tubulus cells. Infect. Immun., 58, 3434-3437.

Messing, J. \& Vieira, J. (1982), A new pair of M13 vectors for selecting either strand of double-digest restriction fragments. Gene, 19, 269-276.

Moch, T., Hoschützky, H., Hacker, J., Kröncke, K.D. \& 
Jann, K. (1987), Isolation and characterization of the $\alpha$-sialyl- $\beta$-2,3-galactosyl-specific adhesin from fimbriated Escherichia coli. Proc. nat. Acad. Sci. (Wash.), 84, 3462-3466.

Morschhäuser, J., Hoschützky, H., Jann, K. \& Hacker, J. (1990), Functional analysis of the sialic-acid-binding adhesin SfaS of pathogenic Escherichia coli by sitespecific mutagenesis. Infect. Immun., 58, 2133-2138.

Orskov, F. \& Orskov, I. (1983), Serology of Escherichia coli fimbriae. Progr. Allergy, 33, 80-105.

Ott, M., Schmoll, T., Goebel, W., Van Die, I. \& Hacker, J. (1987), Comparison of the genetic determinant coding for the S-fimbrial adhesin (sfa) of Escherichia coli to other chromosomally encode fimbrial determinants. Infect. Immun., 55, 1940-1943.

Ott, M., Hoschützky, H., Jann, K., Van Die, I. \& Hacker, J. (1988), Gene clusters for S-fimbrial adhesin (sfa) and F1C fimbriae (foc) of Escherichia coli: comparative aspects of structure and function. J. Bact., 170, 3983-3990.

Parkinen, J., Rogers, G.N., Korhonen, T., Dahr, W. \& Finne, J. (1986), Identification of the O-linked sialyloligosaccharides of glycophorin A as the erythrocyte receptors for S-fimbriated Escherichia coli. Infect. Immun., 54, 37-42.

Riegman, N., Van Die, I., Leunissen, J., Hoekstra, W. \& Bergmans, H. (1988), Biogenesis of $F 7_{1}$ and $F 7_{2}$ fimbriae of uropathogenic Escherichia coli: influence of the FsoF and the FsoG proteins and localization of the Fso/FstE protein. Mol. Microbiol., 2, 73-80.
Riegman, N., Kusters, R., Van Veggel, H., Bergmans, H., Van Bergen en Henegouwen, P., Hacker, J. \& Van Die, I. (1990), F1C fimbriae of a uropathogenic Escherichia coli strain: genetic and functional organization of the $f o c$ gene cluster and identification of minor subunits. J. Bact., 172, 1114-1120.

Sanger, F., Nicklen, S. \& Coulson, A.R. (1977), DNA sequencing with chain-terminating inhibitors. Proc. nat. Acad. Sci. (Wash.), 74, 5463-5467.

Schmoll, T., Hoschützky, H., Morschhäuser, J., Lottspeich, F., Jann, K. \& Hacker, J. (1989), Analysis of genes coding for the sialic acid-binding adhesin and two other minor fimbrial subunits of the S-fimbrial adhesin determinant of Escherichia coli. Mol. Microbiol., 3, 1735-1744.

Schmoll, T., Morschhäuser, J., Ott, M., Ludwig, B., Van Die, I. \& Hacker, J., Nucleotide sequence, regulation and genetic organization of the entire Escherichia coli S-fimbrial adhesin ( $s f a$ ) determinant : functional and evolutionary aspects. Microbial Path. (in press).

Van Die, I., Van Geffen, B., Hoekstra, W. \& Bergmans, H. (1985), Type F1C fimbriae of an uropathogenic Escherichia coli strain : cloning and characterization of the genes involved in the expression of the $1 \mathrm{C}$ antigen and nucleotide sequence of the subunit gene. Gene, 34, 187-196.

Virkola, R., Westerlund, B., Holthofer, H., Parkinen, J., Kekomaki, M. \& Korhonen, T.K. (1988), Binding characteristics of Escherichia coli adhesins in human urinary bladder. Infect. Immun., 56, 2615-2622. 